\title{
Conexões
}

Educação Fisica, Esporte e Saúde

DOI 10.20396/conex.v18i0.8659480

Entrevista

\section{Entrevista a Pere Lavega, heredero de la excelencia intelectual de Pierre Parlebas}

\author{
Aaron Rillo-Albert ${ }^{1}$ \\ Cristòfol Salas-Santandreu ${ }^{2}$ \\ Enirc Ormo-Ribes ${ }^{2}$
}

\section{RESUMEN}

En la entrevista, los autores intentan dar a conocer la vida académica e investigadora de uno de los más importantes discípulos de Pierre Parlebas. Relata sus inicios y posterior profundización en el conocimiento de la praxiología motriz. Su trayectoria investigadora se inicia vinculada inicialmente a los juegos deportivos tradicionales (JDT) y a la etnomotricidad. Actualmente dirige el Grupo de investigación en Acción Motriz (GIAM), con importantes vínculos con Brasil. El grupo se rige por los principios de trabajo en equipo, la praxiología motriz, como fundamentación teórica y la investigación aplicada. Actualmente la línea de investigación intenta aportar evidencias científicas de los efectos de los juegos deportivos sobre las conductas motrices, desde distintas dimensiones de la personalidad. Finalmente destacar que actualmente es presidente de la Asociación Europea de Juegos y Deportes Tradicionales, cargo desde el cual promueve acciones internacionales en red.

Palabras Clave: Parlebas. Praxiologia motriz. Investigación en acción motriz.

\footnotetext{
1 Universidad de Barcelona.

2 Universidad de Lleida.
}

\section{Correspondência:}

Aaron Rillo-Albert. Universidad de Barcelona, National Institute of Physical Education of Catalonia, Gran Via de les Corts Catalanes, 585, 08007 Barcelona, España, Email: arilloalbert@gmail.com 


\section{Entrevista com Pere Lavega, herdeiro da excelência intelectual de Pierre Parlebas}

\section{RESUMO}

$\mathrm{Na}$ entrevista, os autores apresentam a vida acadêmica e de pesquisa de um dos mais importantes discípulos de Pierre Parlebas. Relata o seu início e o subsequente aprofundamento no conhecimento da Praxiologia Motriz. A sua carreira de pesquisador inicia-se vinculada aos jogos esportivos tradicionais (JET) e a etnomotricidade. Atualmente dirige o Grupo de Investigação em Ação Motriz (GIAM), com importantes vínculos com o Brasil. O grupo rege-se pelos princípios do trabalho em equipe, a Praxiologia Motriz, como base teórica e a pesquisa aplicada. Atualmente, a linha de pesquisa tenta aportar evidências científicas dos efeitos dos jogos esportivos sobre as condutas motrizes, a partir de diferentes dimensões da personalidade. Por último, destacar que atualmente Pere Lavega é presidente da Associação Europeia de Jogos e Esportes Tradicionais, cargo a partir do qual promove ações internacionais em rede.

Palavras-chave: Parlebas. Praxiologia Motriz. Pesquisa em ação motriz.

\section{Interview with Pere Lavega, heir to the intellectual excellence of Pierre Parlebas}

\section{ABSTRACT}

In the interview, the authors try to present the academic and research life of one of the most important disciples of Pierre Parlebas. He tells of his beginnings and subsequent deepening of his knowledge of motor praxeology. His research career began linked initially to traditional sports games (TSG) and ethnomotricity. Currently, he directs the Motor Action Research Group (GIAM), with important links to Brazil. The group is governed by the principles of teamwork, motor praxeology as a theoretical foundation and applied research. Currently, the research line tries to provide scientific evidence of the effects of sports games on motor behaviour, from different personality dimensions. Finally, it should be noted that he is currently president of the European Association of Traditional Sports and Games, a position from which he promotes international network actions.

Keywords: Parlebas. Motor Praxeology. Research in motor action. 


\section{CONSIDERACIONES INTRODUCTORIAS}

\section{Pere lavega Burgués}

Licenciado en Educación Física por la Universidad Politécnica de Madrid (INEF Madrid, 1988); Doctor en Filosofía y Ciencias de la Educación por la Universidad de Barcelona (UB, 1995); Catedrático del Instituto Nacional de Educación Física de Cataluña (INEFC), Universidad de Lleida (UdL), especialista en juegos y deportes tradicionales; Actualmente es subdirector de investigación del INEFC, Centro de Lleida; Investigador Principal del Grupo de Investigación en Acción Motriz (GIAM; SGR 2017 197; UdL); Miembro del Grupo de Investigación Technique te Enjeux du corps (Universidad Paris V); Miembro del Instituto de Desarrollo Social y Territorial (INDEST, UdL).

Además, es presidente de la Asociación Europea de Juegos y Deportes Tradicionales (AEJeDT); miembro del Comité Consultivo (Adhoc Advisory Committee) de la UNESCO para la Promoción de los Juegos Tradicionales; vicepresidente y miembro fundador de la Asociación Internacional de Juegos y Deportes Tradicionales (ITSGA) y miembro fundador de la Asociación Internacional de Praxiología Motriz (AIPRAM). Autor de diferentes libros y artículos de impacto sobre juegos, cultura y construcción de las emociones, la cohesión social y la resolución de conflictos. Director de varias tesis doctorales e investigador principal de proyectos europeos.

\section{Pierre Parlebas}

Pierre Parlebas es un pensador, profesor e investigador de incomparable trayectoria en el terreno de la educación física. Motivado por lo que considera una crisis en esta disciplina, investiga, reflexiona y produce hasta sentar los principios que hacen que la acción motriz se constituya como una ciencia con un objeto de estudio propio: la Teoría de la Praxiología Motriz.

A lo largo de toda su vida, su prolífica producción científica, así como la divulgación, han hecho que la Praxiologia Motriz se haya constituido como punto de referencia para el estudio de la motricidad humana. Sus postulados, dan respuesta a todas las preguntas que no podían ser resueltas desde otras ramas de la ciencia y si desde una epistemología específica.

Nacido en Paris en 1934, se inicia como monitor, pasando a entrenador y profesor en las Escuelas Normales de Educación Física, lo que le lleva a interpretar la educación física desde sus cimientos y a ser miembro del Movimiento Nacional de Educación Nueva. Investigador y docente en el INSEP (Institut National du Sport, de I'Expertise et de la Performance), se doctoró en sociología por la universidad de la Sorbona, donde obtuvo la catedra Humanidades. 
Autor de una obra de referencia, Contribution à un lexique commenté en science de l'action motrice (1981), precisa y amplia su aportación en otra gran obra Jeux, sports et sociétés: lexique de praxéologie motrice (1999), todo ello apoyado por una amplísima producción científica.

Actualmente continúa su labor como divulgador y conferenciante. Doctor Honoris causa por varias universidades, le fue otorgado el grado de Caballero de la Legión de Honor francesa por sus logros en la docencia, divulgación e investigación en la Praxiología Motriz.

A continuación, se presenta una entrevista especial para la revista Conexões: Educação Física, Esporte e Saúde.

Pere Lavega ha sido uno de los principales seguidores de Pierre Parlebas desde que descubrió sus aportaciones en el campo de la educación física, planteándose una nueva manera de entender esta disciplina, una educación física moderna. En esta entrevista, el Dr. Lavega habla del cambio de paradigma y de la necesidad de "desaprender para volver a aprender". Durante todos estos años, ha estudiado, interpretado, investigado, divulgado y sigue descubriendo todo aquello que engloba la teoría de la praxiología motriz.

\section{AUTORES: ¿CUÁLES FUERON SUS PRIMEROS CONTACTOS CON LA PRAXIOLOGÍA MOTRIZ?}

Dr. Lavega: todo empezó en el año 1985, en una clase de la asignatura de Sociología del Deporte, con el profesor Paco Lagardera, cuando nos presentó por primera vez unos apuntes traducidos al español de Antonio Galera sobre la clasificación de las prácticas motrices que hace Pierre Parlebas. En aquel momento, Paco nos explicó una nueva teoría que rápidamente me hizo replantearme muchas cosas. Este descubrimiento, despertó la curiosidad e inquietud por ir más allá del recetario que a menudo había visto durante la etapa universitaria. La ciencia de la acción motriz me obsequiaba con nuevos planteamientos; en esos momentos éramos estudiantes, muy jóvenes y esta nueva manera de entender nuestro ámbito nos aportaba una revolución intelectual, un cambio de perspectiva, un nuevo paradigma.

\section{AUTORES: ¿QUÉ IMPACTO TUVO A CORTO PLAZO LA NUEVA PERSPECTIVA PLANTEADA POR LA PRAXIOLOGÍA MOTRIZ?}

Dr. Lavega: la ciencia de la acción motriz en seguida me cautivó y me permitió alcanzar varios hitos académicos y profesionales. Siendo estudiante de quinto curso de licenciatura, me destinaron a Madrid tras olvidar pedir una prórroga militar. Por lo tanto, me trasladé a esta ciudad donde hice el servicio militar y también terminé mi carrera universitaria tras haber solicitado el traslado de expediente de Lleida a esa ciudad. La primera contribución de la praxiología motriz, fue el uso de unos apuntes de Pierre, en un examen teórico de la asignatura de Fundamentos de la 
Táctica Deportiva. El resultado fue magnífico, me concedieron matrícula de honor.

Posteriormente, fruto de las circunstancias apareció la opción de presentarme a una plaza de profesor contratado temporalmente en el Instituto Nacional de Educación Física de Cataluña (INEFC), en las asignaturas de deportes (fútbol) y de Juegos aplicados a la Educación Física. En ese concurso competitivo, presenté un proyecto fundamentado en los apuntes y otros materiales de Pierre Parlebas, de la praxiología motriz. El planteamiento gustó al tribunal y finalmente conseguí el contrato de profesor asociado en el INEFC de Lleida.

\section{AUTORES: ASÍ PUES, SUS INICIOS COMO PROFESOR UNIVERSITARIO SE DAN TANTO EN EL FÚtBOL COMO EN EL ÁMBITO DE LOS JUEGOS APLICADOS, ¿POR QUÉ SE DECANTÓ POR ESTE SEGUNDO ÁMBITO?}

Dr. Lavega: al cabo de un par de años tuve que tomar la decisión de optar por al área de fútbol o dedicarme al ámbito de juegos. No fue una decisión fácil; la praxiología motriz me ayudaba a identificar dos escenarios totalmente diferentes. Optar por la plaza de ser profesor del deporte del futbol, del cual soy un entusiasta, suponía acotar el campo de acción a un solo deporte. En cambio, si me decantaba por el ámbito del juego me encontraría ante un universo de acción muy variado de tipología de actividades. La decisión no fue fácil, teniendo en cuenta que yo accedí a estos estudios en INEFC tras haber sido jugador de fútbol. Finalmente me decanté por el ámbito del juego y terminé consolidando mi plaza; primero como profesor titular y posteriormente como catedrático. Esta primera fase como docente, la praxiología motriz despertó en mí muchas reflexiones profundas en torno a nuestra profesión y nuestra labor universitaria.

\section{Autores: ¿En Qué momento conoce Personalmente a Pierre Parlebas?}

Dr. Lavega: en 1990 decido iniciar el programa de doctorado en el único programa que existía en aquel entonces en Cataluña. Durante dos años me desplacé regularmente a Barcelona para asistir a las clases presenciales. Como alumno de doctorado tuve la suerte de conocer personalmente a Pierre Parlebas, quien me sorprende y me fascina. Me dedicó su libro Elementos de Sociología del Deporte (1988) (primera edición) y me confirmó que a veces es necesario desaprender para aprender y encontrar el orden buscado. En aquella época en 1991 nace el Grupo de Estudios Praxiológicos (GEP) que ha ido evolucionado hasta llegar al momento actual.

\section{AUTORES: ¿PORQUE DESAPRENDER?}

Dr. Lavega: en mi condición de estudiante, la formación recibida correspondía principalmente a una concepción de la educación física orientada a cuestiones de ejecución, de habilidad, del movimiento mecánico. Desconocía aportaciones que me permitieran comprender que la clave está en desvelar las relaciones o el 
significado de las acciones motrices. El salto, es muy distinto si se efectúa al tirarse a una piscina, al hacer un salto de longitud, al saltar para contactar con un adversario en taekwondo, o al hacer un remate de voleibol. Si hablamos de fuerza, velocidad o potencia de salto, dejamos de lado ese contexto relacional y de significado que sirve para desvelar el sentido de cualquier salto. El desafío es ir más allá de nociones superficiales, abandonar el paradigma instalado en el "movimiento" y las leyes físicas y mecánicas, superar el enfoque energético y abrir la puerta a otra manera de comprender las prácticas motrices. En las primeras promociones de INEFC, los estudiantes estábamos fuertes físicamente, las pruebas físicas eran muy exigentes y el dominante energético era un denominador común del día a día. Recuerdo haber estudiado con detalle el libro de Álvarez-Del Villar: "La preparación física del futbol basada en el atletismo", ienergía, energía y energía! Este ejemplo permite ver el error de base, al pretender provocar transferencia de acción entre dos deportes (atletismo y fútbol) que tienen una lógica motriz muy distinta, ya que sus mecanismos de funcionamiento tienen una naturaleza distinta.

Además, necesitamos dignificar nuestra profesión y el "chándal"; necesitamos dotarnos de un conocimiento específico y original y también poder mostrar a compañeros científicos de otras facultades, que nosotros, desde la educación física también podemos investigar y generar conocimiento científico basado en evidencias.

Cuando escucho a Pierre Parlebas en el curso de doctorado, me hace movilizar todas estas inquietudes. Cada frase y reflexión es una sentencia contundente apoyada en evidencias científicas. Cada afirmación no hace más que dar consistencia a nuestra profesión y a nuestras intervenciones en los distintos ámbitos de acción.

\section{Autores: ENTONCES, CUANDO SURGE LA NECESIDAD DE INVESTIGAR DESDE LA ESPECIFICIDAD NACE EL GEP.}

Dr. Lavega: en el 1991 nació nuestro grupo de investigación con mucha humildad. Un grupo reducido de profesores noveles empezamos a encontrarnos y a intentar compartir todos los materiales que nos llegaban de Pierre Parlebas. Al cabo de dos años, en 1993, nos atrevimos a coordinar y publicar un monográfico, en la revista Apunts. Educación Física y Deportes. En mi opinión, nos precipitamos, todavía no estábamos preparados para comprender profundamente las bases científicas de la praxiología motriz; pienso que cometimos un error. Si ahora pudiera volver atrás en el tiempo, borraría ese "lunar" de nuestra historia. Sin embargo, en ese grupo estábamos dispuestos a trabajar intensamente, la ilusión y las ganas de aprender eran nuestra tarjeta de presentación. En ese contexto de gran ebullición emocional, en el año 1992, empecé mi tesis doctoral. 


\section{Autores: ¿Quién era el InVestigador Principal (IP) Del grupo de INVESTIGACIÓN POR AQUÉL ENTONCES?}

Dr. Lavega: el Dr. Lagardera era el IP del grupo, él había sido nuestro profesor de sociología durante la licenciatura. Gracias a su formación erudita, iba desvelando la contribución de la praxiología motriz, su fundamentación en la noción de estructura y en la teoría de sistemas. En esa etapa, estábamos inmersos en intentar conocer los fundamentos que todavía no dominábamos en toda su magnitud.

\section{AUTORES: FINALIZADAS LAS ASIGNATURAS DE DOCTORADO, FUE EL MOMENTO DE ELEGIR EL OBJETO DE ESTUDIO DE LA TESIS DOCTORAL Y TAMBIÉN DIRECTOR DE TESIS ¿CóMO FUE ESE PROCESO?}

Dr. Lavega: tras terminar los dos años del programa de doctorado, necesitaba un tema que investigar y también un director de tesis. Así que me puse en contacto con diferentes personas; recuerdo haber contactado con el Dr. Josep Roca, (psicólogo de gran prestigio) y también con diversos catedráticos, como el profesor Llorenç Prats especialista en historia. La respuesta se repetía en todos los casos: debía hacer una tesis fuera del ámbito de la educación física, ejerciendo el rol de psicólogo o historiador. Mi prioridad era hacer una tesis que después pudiera tener una aplicación directa al ámbito del juego. En aquel momento, tuve la suerte que Paco Lagardera defendió su tesis doctoral en el INEFC de Barcelona, de manera que, a la semana siguiente, ya con el reciente título de Doctor, le pedí que fuera mi director de tesis. Ya tenía director de tesis y también objeto de estudio: la interpretación del proceso de transformación en deporte de un juego tradicional, los bolos ("bitlles" en catalán) muy arraigado a las tierras de Lérida. Era un juego que estaba en plena transformación y me iba permitir profundizar en la perspectiva etnomotriz, es decir, en el análisis de su lógica interna y también de aspectos socioculturales de la cultura local.

\section{AUTORES: ¿Cómo RECUERDA ESE PERÍOdO DE REALIZACIÓN DE LA TESIS DOCTORAL?}

Dr. Lavega: en el año 1992 empecé el cargo de subdirector de estudios y recuerdo que Paco Lagardera consideraba que no podría asumir ambos desafíos al mismo tiempo, sugiriéndome que dejara este nuevo cargo directivo. Convencido de que podría alcanzar ambos objetivos, me impuse una disciplina espartana, levantándome muy pronto todos los días, destinando un día entero a la semana (los lunes) a la tesis doctoral. Todo esto me permitió en el 1995 antes de terminar el cargo, entregar la tesis doctoral. El 16 de octubre de 1995 (un lunes) se hizo el acto de lectura de tesis en Barcelona. Recuerdo que estuve todo el fin de semana en Barcelona probando todos los materiales y preparando esa defensa de la tesis.

\section{Autores: en el tribunal de su tesis doctoral estuvo el Dr. Pierre Parlebas.}

Dr. Lavega: en el año 1994, fui a París aprovechando que mi amigo Joseba 
Etxebeste estaba disfrutando de una estancia de investigación. El viaje me permitió conocer a Pierre Parlebas en otro contexto menos formal y proponerle que formara parte del tribunal de mi defensa de tesis doctoral. El trato fue muy cordial y próximo. Me enseñó su despacho, su laboratorio; fue una experiencia casi mágica, poder subir por unas escaleras y acudir a su despacho en la Sorbona de París, la catedral mundial de las ciencias sociales.

El día de la defensa de tesis me hizo mucha ilusión ver la sala de grados, llena de gente, con amigos que habían venido de diferentes lugares, porque claro, en el tribunal estaban Dr. Pierre Parlebas, Dr. J. Hernández-Moreno, Dr. J. Petrus y el Dr. C. Feixa.

También aprovechamos para hacer el primer seminario el día siguiente porque después había un congreso que organizaban en aquella época cada dos años al INEFC de Lérida. Por lo tanto, no era solo por la tesis, sino que por este conjunto de cosas había un grupo de compañeros de diferentes lugares de España, Francia, Portugal y Túnez que hizo que el acto fuera bastante singular. El acto duró 3 horas y fue algo muy excepcional.

\section{AUTORES: ¿CÓMO FUE ESE PRIMER SEMINARIO INTERNACIONAL?}

Dr. Lavega: al día siguiente, del 17 de octubre de 1995 tuvo lugar el primer seminario internacional. Empezamos a debatir aspectos relacionadas con la tesis y después otros temas e inquietudes que compartíamos: Parlebas, During, Collard, Hernández Moreno, Castro, Etxebeste, Elloumi, Lagardera, etc., estaban presentes. Al día siguiente empezó nuestro congreso (INEFC Lleida) con aportación de comunicaciones en la sección de la ciencia de la acción motriz. Ese seminario en Lleida supuso el inicio de los seminarios internacionales, de modo que empezamos a entrar en contacto con otros grupos de España, Francia, Túnez y Portugal. Tras comprender el sentido y profundidad de los documentos de Parlebas, vimos la necesidad de traducir al español la obra principal de Pierre Parlebas. Juegos, deporte y Sociedad. Léxico comentado de praxiología motriz (2001). Paco Lagardera, coordinó esta acción y durante un año estuvimos trabajando intensamente con traductores de la editorial Paidotribo.

Acto seguido decidimos elaborar un texto divulgativo, Introducción a la Praxiologia Motriz (2003) con la editorial Paidotribo. Igualmente, participamos en la revisión, modificación y mejora de la obra Elementos de Sociología del Deporte (2003) que no estaba muy bien traducida al español en su primera edición.

\section{Autores: Y en 2002 Se nombra doctor Honoris Causa Al Dr. PierRe Parlebas POR LA UNIVERSIDAD DE LLEIDA.}

Dr. Lavega: tras participar en el seminario de praxiología motriz en Madrid, en la estación de tren preparados para volver a Lérida, Paco Lagardera y yo 
compartimos la idea de solicitar la distinción a Pierre Parlebas, como doctor honoris causa por la Universidad de Lleida. Tras presentar ese proyecto en nuestro centro y en la Universidad de Lleida, en el año 2002 se hizo realidad ese reconocimiento. Por primera vez a nivel internacional y a nivel estatal, una persona de nuestro ámbito recibía esta distinción por sus méritos científicos. Ese acto permite proyectar a nivel nacional e internacional el estatus científico del INEFC, de la actividad física y el deporte y de nuestra profesión.

\section{AUTORES: TODA ESTA TRAYECTORIA INICIAL DE INVESTIGACIÓN LE LLEVA A OBTENER LA PLAZA DE CATEDRÁTICO EN EL INEFC DE LLEIDA.}

Dr. Lavega: transcurridos unos años, en 2005 consolidé la plaza de catedrático; el proyecto que defendí ante un tribunal se basó en la aplicación de la praxiología motriz desde una perspectiva etnomotriz, es decir, relacionando el juego, la educación y la cultura.

\section{AUTORES: ¿NOS PODRÍA INDICAR CUÁL HA SIDO EL ITINERARIO SEGUIDO EN EL OBJETO DE ESTUDIO DE LAS INVESTIGACIONES?}

Dr. Lavega: para entender mi itinerario intelectual debemos partir de una idea clave comprender lo que nos aporta esta disciplina, es decir todo gira en torno a desvelar la relación entre el sistema que representa cualquier juego, deporte o práctica motriz y sus efectos sobre el actor, el jugador, el alumno, la persona que juega, que entrena, que está en un centro de vacaciones, etc., en este caso a través de conductas motrices.

El estudio del sistema: la lógica interna. En la primera etapa de mis investigaciones, centro la atención en el estudio del sistema, es decir, de la lógica interna de los juegos. Centro la atención en el estudio monográfico de un juego tradicional, los bolos desde la etnomotricidad, para interpretarlo en relación con la cultura local. Este proceso me da suficiente bagaje para posteriormente estudiar desde una perspectiva etnomotriz los juegos tradicionales en Cataluña. Eso fue posible, gracias a diversas becas concedidas por el departamento de cultura, a través de su centro de promoción de la cultura popular y tradicional. Realizamos un inventario, un estudio etnomotor y empezamos a desvelar la contribución de este conjunto de prácticas en el campo educativo, consideradas a menudo minoritarias o secundarias. Encontramos que favorecen valores de máxima actualidad para una educación física moderna, a modo de ejemplo destaco: a) las relaciones interpersonales (los juegos son sobre todo sociomotores); b) competir y compartir; existe igualdad de proporción de juegos con y sin competición asociada a un marcador; c) la sostenibilidad: los juegos emplean objetos que se reciclan del entorno y también se puede jugar sin objetos; d) la igualdad de oportunidades: se identifican juegos masculinos, femeninos y mixtos, las personas de todas las edades o condición social pueden intervenir. 
El estudio de las conductas motrices. Todo ello, nos lleva a una segunda etapa, más complicada y atractiva ya que significa acudir al terreno de juego, el gimnasio, el pabellón o el patio para estudiar los efectos, en este caso sobre el actor, sobre sus conductas motrices. Es entonces cuando con el cambio del plan de estudios de la licenciatura, se incorpora una asignatura que considera una familia de juegos poco atendida como son los juegos cooperativos. Me ofrezco voluntario para impartir dicho contenido y seguidamente pasamos a realizar una identificación; un catálogo de conductas motrices cooperativas. Paralelamente el profesor Paco Lagardera hace lo mismo sobre las conductas motrices introyectivas.

Posteriormente vinieron las tesis doctorales, trabajos de investigación con miembros del grupo vinculados con temas de conflictos con Unai Sáez de Ocáriz; sobre la cohesión grupal Juan Andueza; la toma de decisiones y estados emocionales Jorge Serna. Además, en este caso iniciamos un camino importante que Pierre Parlebas abrió en la década de los sesenta, una de las claves en el mundo de la educación, la afectividad. Dado que no se había iniciado ningún trabajo en esa área, en 2007 decidimos activar esta línea de investigación, contactando con otro grupo de investigación de la universidad de Lleida: el GROP (Grupo de Investigación en Orientación Psicopedagógica) especializado en la educación emocional. En el año 2009 conseguimos que el Ministerio de Ciencia y Educación de España nos conceda un proyecto de investigación coordinado. Investigamos los efectos de los distintos dominios de acción motriz sobre los estados emocionales. Participan compañeros de diversas regiones de España y de Portugal. Y también se añaden otros compañeros de Suiza y de Brasil, entre los que menciono Marco Bortoleto y Joao Ribas. Empezamos ese nuevo camino sobre el que estamos avanzando en estos momentos.

Tras unos años de estudios empíricos, ahora tenemos entre manos un gran desafío, desvelar los efectos de los juegos deportivos sobre las conductas motrices, desde distintas dimensiones de la personalidad; es una mirada $360^{\circ}$ al intentar poner al descubierto al mismo tiempo la significación desde un punto de vista orgánico, cognitivo, relacional y emocional.

\section{AUTORES: ¿QUÉ OTROS HITOS DESTACARÍAN EN SU TRAYECTORIA COMO INVESTIGADOR?}

Dr. Lavega: el año 2017 inicio dos experiencias muy especiales. Soy elegido presidente de la Asociación Europea de Juegos y Deportes Tradicionales, y también soy invitado a formar parte de un grupo de expertos de la UNESCO. Se crea un comité consultivo adhoc para la promoción y la salvaguarda de los juegos tradicionales. Me encargo de coordinar un grupo de trabajo para establecer directrices políticas para la salvaguarda de los juegos deportivos tradicionales (JDT) y esto me permite entrar en contacto con mucha gente de alrededor del mundo.

Todo esto me estimula para activar acciones internacionales en red. Así que en el 
año 2017 presentamos el proyecto europeo Bridge a la convocatoria Erasmus+: cuatro institutos de España, Portugal, Francia e Italia junto con cuatro universidades que como indica su nombre (Bridge, es decir puente) comparten una experiencia educativa mediante el uso de juegos tradicionales para favorecer la convivencia, el diálogo intercultural entre personas de diferentes países. El proyecto culmina con un encuentro en 2018 en Verona donde coinciden estudiantes de INEFC, profesores de diferentes universidades, alumnos y profesores de educación física de estos países y también se cuenta con la presencia de Pierre Parlebas. Así se hace realidad llevar la teoría a la práctica en distintos países.

En el año 2019 iniciamos otro proyecto europeo Erasmus+. El proyecto lo denominamos Together y supone dar un paso más, en el terreno de la inclusión social de personas con discapacidad intelectual mediante los JDT. Ahora en 2020 acabamos de presentar un proyecto de gran envergadura en torno a la inclusión social y la igualdad de género con la participación de 9 socios y 7 países.

En todas estas iniciativas, la teoría y la práctica son un binomio inseparable. Además, nos interesa acercarnos al profesional que trabaja en el propio terreno de acción, inquietud que nos lleva a elaborar materiales aplicados. De ahí que hayamos organizado dos cursos online MOOC (massive open on-line courses), innovadores, en torno al tema aprender a convivir a través del juego. Las cuatro ediciones del curso I y la primera edición del curso II ha permitido contar con más de 7000 personas inscritas de 48 países.

\section{AUTORES: ¿QUÉ CARACTERÍSTICAS SUBRAYARÍA DE SU GRUPO DE INVESTIGACIÓN EN ACCIÓN MOTRIZ (GIAM)?}

Dr. Lavega: para nosotros investigar es una actitud (trabajar en equipo) que debe ir acompañada de una fundamentación científica (la praxiología motriz). La ciencia de la acción motriz nos marca el camino para plantearnos el objeto de estudio de las investigaciones, para identificar las variables independientes y dependientes, así como para establecer diferentes estrategias metodológicas en los estudios.

Para mi investigar es trabajar en equipo, compartiendo experiencias, reflexiones y aportaciones de personas con gran curiosidad por generar conocimiento científico. Todas esas personas tienen oportunidad de intervenir en equipo a pesar de tener diferentes niveles de formación: estudiantes (de grado, máster o doctorado), profesores (jóvenes o expertos), becarios, etc. Desde nuestra creación en 1991 nos reunimos una vez al mes; recuerdo que hace años algunos compañeros empleaban hasta tres horas de viaje para asistir a las reuniones del grupo. Ahora la tecnología nos permite compartir esas reuniones con compañeros de España, Brasil, Portugal o Túnez por videoconferencia. En las últimas reuniones estamos participando unas 20 personas. 


\section{AUTORES: EN LOS GRUPOS DE INVESTIGACIÓN QUE HAS LIDERADO, HA HABIDO DIFERENTES CAMBIOS DE DENOMINACIÓN DEL NOMBRE DEL GRUPO, ¿PODRÍAS EXPLICAR CÓMO VA CAMBIANDO LA DENOMINACIÓN DEL GRUPO Y POR QUÉ?}

Dr. Lavega: inicialmente nacemos como grupo de estudios praxiológicos (GEP). Paco Lagardera es el alma del grupo y el investigador principal. Cuando Paco por edad, con motivo de su prejubilación deja el liderazgo del grupo, yo asumo el relevo. En aquel momento, se activa el reconocimiento oficial de los grupos de investigación del INEFC, por parte de AGAUR, la agencia que gestiona las ayudas de investigación en el sistema de universitario de Cataluña. En aquel momento, con los miembros del grupo trabajábamos muy intensamente en el área de los Juegos deportivos decidimos denominar el grupo GREJE (Grupo de investigación en juegos deportivos).

Hacia el año 2010 se incorporan al grupo varios profesores del Centro que trabajan en diferentes ámbitos de acción: la didáctica, el entrenamiento, el baloncesto, el balonmano, el voleibol, la gimnasia rítmica, la natación, las actividades en la naturaleza, los juegos tradicionales, los conflictos, los juegos tradicionales, el uso de las nuevas tecnologías... Por ese motivo, y con la intención de que todos se sientan plenamente integrados, se decide un nuevo nombre: grupo de investigación en acción motriz (GIAM).

\section{Autores: ¿CuÁl es su Relación actual con PierRe Parlebas?}

Dr. Lavega: la relación con Pierre ha pasado por distintas etapas; primero como alumno (del programa de doctorado y de cursos que impartió en España). Tras defender la tesis doctoral inicié una segunda etapa de amigo que se consolidó con motivo de la investidura como doctor honoris causa en 2002. A partir de entonces nuestra amistad ha ido en aumento; he estado en su segunda residencia en Sant León, lugar donde nació y he compartido un buen número de viajes y experiencias en congresos, encuentros de la UNESCO y proyectos internacionales. En todas esas ocasiones, las conversaciones con Pierre son un tesoro que forma parte de mi experiencia vital. Me considero una persona privilegiada por haber conocido en persona, al Einstein, al Darwin, al Freud o al Piaget de la educación física. A sus 86 años sigue dando lecciones magistrales cuando imparte una conferencia o improvisa una reflexión sobre cualquier tema de la vida o de naturaleza científica.

\section{AUTORES: ¿PARA FINALIZAR NOS GUSTARÍA SABER QUÉ CONEXIONES TIENE USTED CON LOS GRUPOS DE PRAXIOLOGÍA MOTRIZ DE BRASIL?}

Dr. Lavega: para comprender la trayectoria de nuestro grupo es necesario reconocer las aportaciones de todos los compañeros que han pasado desde que su creación. De entre estos investigadores merece una atención especial el profesor Dr. Joao Francisco Magno Ribas de la Universidad de Santa María de Rio Grande do Sul. Joao vino inicialmente a nuestro Centro gracias a un programa de movilidad de 
profesores de Latinoamérica que se llamaba Intercampus. Posteriormente realizó su tesis doctoral con nosotros, aunque con un programa de doctorado de Brasil. De vuelta a Brasil animó a sus compañeros, a ex-alumnos a que viajaran hasta nuestro centro. Así el profesor Marco Antonio Coelho Bortoleto, cursó el programa de doctorado y defendió su tesis con nuestro grupo. Posteriormente le han seguido otros compañeros de esa zona de Brasil: Pablo Aires, Marcia Filipetto, Silvester Franchi, Sabrine Damian y Leonardo Machado. Por este motivo la historia de GIAM se escribe en "portugués" de Brasil.

Esta relación también nos ha permitido compartir seminarios en Brasil y Argentina, además de impulsar actos tan importantes como la distinción de Parlebas como doctor honoris causa por la Universidad de Campinas.

AUTORES. Tras una hora y media de conversación finalizamos esta entrevista con Pere Lavega, coordinador de GIAM, discípulo de Paco Lagardera y adicto a la excelencia intelectual y personal que le ha regalado Pierre Parlebas $y$ el conocimiento de su obra.

\section{REFERENCIAS}

LAGARDERA, Francisco; LAVEGA, Pere. Introducción a la Praxiología motriz. Barcelona: Editorial Paidotribo, 2003.

PARLEBAS, Pierre. Contribution à un lexique commenté de l'action motrice. Paris: INSEP, 1981.

PARLEBAS, Pierre. Elementos de sociología del deporte. Málaga: Universidad Internacional Deportiva de Andalucía, 1988.

PARLEBAS, Pierre. Jeux, sport et sociétés: lexique de praxéologie motrice. Paris: INSEP, 1999.

PARLEBAS, Pierre. Juegos, deporte y sociedades. Léxico de praxiología motriz. Barcelona: Editorial Paidotribo, 2001.

PARLEBAS, Pierre. Elementos de Sociología del Deporte. Málaga: Instituto Andaluz del Deporte, 2003. 